\title{
The Cholinergic System and Inflammation: Common Pathways in Delirium Pathophysiology
}

\author{
Joaquim Cerejeira, MD, "广 Vasco Nogueira, MD, "广 Pedro Luís, MD, "Adriano Vaz-Serra, PhD, and \\ Elizabeta B. Mukaetova-Ladinska, MD, PhD
}

OBJECTIVES: To investigate whether delirium is associated with an unbalanced inflammatory response or a dysfunctional interaction between the cholinergic and immune systems.

DESIGN: Cohort observational study.

SETTING: General hospital orthopedic ward.

PARTICIPANTS: One hundred one individuals aged 60 and older with no previous cognitive impairment undergoing elective arthroplasty.

MEASUREMENTS: Incidence of postoperative delirium, plasma cholinesterase activity (acetylcholinesterase (AChE) and butyrylcholinesterase $(\mathrm{BuChE}))$ and inflammatory mediators (C-reactive protein (CRP), interleukin (IL)-1 beta, tumor necrosis factor alpha, IL-6, IL-8, IL-10) before and after surgery.

RESULTS: Thirty-seven participants developed postoperative delirium and had greater production of CRP and proinflammatory to anti-inflammatory ratio after surgery. In participants with delirium, but not in controls, preoperative levels of plasma cholinesterase activity correlated with $\Delta$ CRP (AChE: $\rho=0.428, P=.008$ and BuChE: $\rho=0.423$, $P=.009), \Delta \mathrm{IL}-6$ (AChE: $\rho=0.339, P=.04)$, and $\Delta \mathrm{P} / \mathrm{A}$ ratio (AChE: $\rho=0.346, P=.04$ ).

CONCLUSION: Delirium was associated not only with an unbalanced inflammatory response, but also with a dysfunctional interaction between the cholinergic and immune systems. Comprehensive understanding of the relationship between the cholinergic and immune systems is crucial to developing new insights into delirium pathophysiology and novel therapeutic interventions. J Am Geriatr Soc 2012.

Key words: delirium; inflammation; cholinesterases; C-reactive protein; cytokines

From the "Department of Psychiatry, Hospitais da Universidade de Coimbra, "Faculty of Medicine, Universidade de Coimbra, Coimbra, Portugal; and "Institute for Ageing and Health, Newcastle University, Newcastle upon Tyne, UK.

Address correspondence to Joaquim Manuel Soares Cerejeira, Serviço de Psiquiatria, Hospitais da Universidade de Coimbra, 3000-377 Coimbra, Portugal. E-mail: joaquim.cerejeira@huc.min-saude.pt

DOI: $10.1111 / \mathrm{j} .1532-5415.2011 .03883 . \mathrm{x}$
$\mathrm{D}$ elirium is a common neuropsychiatric syndrome characterized by sudden onset of impaired consciousness and inattention with subsequent cognitive and behavioral disturbance. ${ }^{1}$ From a pathophysiological perspective, this syndrome can be best conceptualized as a dysfunction in the homeostatic response to a precipitating factor (e.g., medical or surgical condition) resulting in an acute failure of the central nervous system (CNS). The two dominant theories regarding delirium pathophysiology are cholinergic deficiency and aberrant stress response and neuroinflammation. $^{2}$

Surgery (e.g., hip arthroplasty) is a controlled traumatic event that directly induces tissue ischemia and destruction amid a diversity of factors such as hypotension, hypoxia, pain, blood loss, anesthetics, and drugs. Globally, the effect of these factors on the body elicits a number of intricate physiological responses characterized by the production and release of proinflammatory cytokines into the bloodstream, activation of the inflammatory cascade, and recruitment of immune cells. Protective immunity is critically dependent on adequate balance between pro- and anti-inflammatory responses to allow proper repair of tissue damage and to combat overwhelming infection without compromising organ function. Thus, counterregulatory mechanisms are simultaneously activated to inhibit the systemic innate immune response. These consist of anti-inflammatory cytokines (e.g., interleukin (IL)-10), stress hormones, and signals originating from the CNS culminating in the release of acetylcholine by the vagus nerve, which interacts with nicotine acetylcholine receptors in immune cells. ${ }^{3}$ Disruption of these complex homeostatic mechanisms may be involved in delirium pathophysiology as increased secretion of proinflammatory cytokines into the bloodstream triggers an exaggerated neuroinflammatory reaction, affecting synaptic and neuronal function and coupled with cognitive and behavioral symptoms similar to delirium. ${ }^{2}$

The few clinical studies conducted so far exploring the inflammatory response in delirium have provided contradictory results. Although high levels of C-reactive protein 
(CRP) have been associated with delirium in medical ${ }^{4-6}$ and surgical ${ }^{6}$ patients, other studies have not supported these findings. ${ }^{7-11}$ Likewise, negative findings ${ }^{8-10,12-14}$ have followed positive associations between delirium and higher plasma levels of IL-6, ${ }^{4-6}$ IL-8, ${ }^{12,15}$ and IL-10. ${ }^{7}$ High serum cytokines have also been found in sickness behavior independently of delirium occurrence (e.g., IL-6 and TNF- $\alpha)^{16}$ or postoperatively in individuals with impaired cognition. ${ }^{17}$

Thus, the relationship between the absolute levels of serum inflammatory mediators and delirium is inconsistent, heterogeneous, and difficult to generalize, compromising their clinical utility as biomarkers for this clinical syndrome. This also confirms that we are far from understanding to what extent the presence of inflammatory mediators in the bloodstream contributes to the development of neuropsychiatric symptoms leading to delirium. Additionally, although the cholinergic antiinflammatory pathway has been well characterized in animal models ${ }^{18}$ and implicated in inflammatory and infectious disorders, ${ }^{3}$ there is a lack of studies analyzing the interconnection between the cholinergic system and the innate immune response in individuals with delirium. A recent study demonstrated that individuals who develop postoperative delirium have substantially lower plasma cholinesterase activity before surgery, suggesting that an altered cholinergic function in resting conditions is a risk marker of delirium. ${ }^{19}$

The current study built on these findings and sought to clarify whether delirium is associated with an unbalanced inflammatory response or a dysfunctional interaction between the cholinergic and immune systems. It specifically addressed whether individuals with delirium would have an imbalance of proinflammatory to antiinflammatory cytokine concentration before or after surgery and a dysfunction in baseline plasma cholinesterases activity influencing the magnitude of the innate immune response to surgery.

\section{MATERIALS AND METHODS}

\section{Preoperative Assessment}

The study included consecutive individuals aged 60 and older undergoing elective total hip arthroplasty and not fulfilling the Diagnostic and Statistical Manual of Mental Disorders, Fourth Edition, Text Revision, (DSM-IV-TR) criteria for dementia or delirium at the time of their preoperative medical evaluation, which included collection of demographic and clinical data and an assessment using the Charlson Comorbidity Index, Barthel Index, Mini-Mental State Examination (MMSE), and 15-item Geriatric Depression Scale.

\section{Medical and Surgical Procedures and Perioperative Factors}

Nonsteroidal anti-inflammatory and antiplatelet drugs were discontinued for at least 1 week before surgery, and thromboprophylaxis (subcutaneous low-molecular-weight heparin) was administered to each participant daily during that week until the day before surgery, with prophylactic intravenous antibiotherapy being initiated on the day of surgery. General anesthesia was induced with propofol, and after the administration of a muscular relaxant, patients were intubated and mechanically ventilated. Alternatively, regional anesthesia (administration of analgesic medication (e.g., levobupivacaine) into the subarachnoid space through a catheter) was used. All patients were monitored during surgery, with fluid loss being replaced using an intravenous crystalloid solution and, if necessary, transfusion of red blood cells or whole blood. A lateral surgical approach with trochanteric osteotomy was used for the arthroplasty.

\section{Postoperative Assessment}

After surgery, all participants were assessed for delirium using the Confusion Assessment Method (CAM) (performed by a trained psychiatrist) on 3 consecutive days between 6 and 8 p.m. The first assessment occurred on the evening of the surgery day and the second and third took place on postoperative days 2 and 3. The CAM was rated during a brief interview with the participant that also included the MMSE. Positive cases of delirium (according to the CAM criteria) were confirmed according to the DSM-IV-TR criteria. The final sample consisted of 101 participants, 37 of whom developed delirium during the study period. Participants with delirium did not differ from those without on any preoperative or perioperative variable, except that they were less likely to consume alcohol regularly (Table 1$)$.

\section{Measurements of Serum Cholinesterase Catalytic Activities}

Venous blood samples were collected from each participant in the morning of the day before surgery and in the morning of the first postoperative day. Plasma was immediately separated by centrifugation and stored at $-80^{\circ} \mathrm{C}$ until analysis. Plasma acetylcholinesterase (AChE) and butyrylcholinesterase $(\mathrm{BuChE})$ activity was assayed by measuring the production of thiocholine from the hydrolysis of the respective specific substrates acetylthiocholine iodide and S-butyrylthiocholine iodide. The production of 5-thio2-nitrobenzoate was monitored using a Cary 100 Spectrophotometer (Varian, Cary, NC) at $412 \mathrm{~nm}$ over time (every minute for 8 minutes). Enzyme activity is expressed as $\mu \mathrm{mol}$ of $5,5^{\prime}$-dithiobis-(2-nitrobenzoic acid) transformed per $\mathrm{mL}$ of plasma per minute. ${ }^{19}$

\section{Measurement of Inflammatory Mediators}

The concentrations of five cytokines (IL-8, IL-1 beta ( $\beta$ ), IL-6, IL-10, tumor necrosis factor alpha (TNF- $\alpha)$ ) and CRP were determined using the xMAP Bio-Plex Suspension Array System 200 with multiplex assay kits purchased from Bio-Rad (Hercules, CA) and Invitrogen (Carlsbad, CA). Standard curves were constructed for determination of each analyte concentration according to the manufacturers' instructions. The detection limits of the assays were $0.002 \mathrm{ng} / \mathrm{mL}$ for CRP, $0.8 \mathrm{pg} / \mathrm{mL}$ for IL- $1 \beta, 3 \mathrm{pg} / \mathrm{mL}$ for TNF- $\alpha, 1.1 \mathrm{pg} / \mathrm{mL}$ for IL- $6,0.5 \mathrm{pg} / \mathrm{mL}$ for IL- 8 , and $0.9 \mathrm{pg} / \mathrm{mL}$ for IL-10. In accordance with standard practice, samples with undetectable cytokine levels were entered at half of the minimum detection level derived from the 
Table 1. Characteristics of the Analyzed Sample

\begin{tabular}{|c|c|c|c|c|}
\hline Characteristic & $\begin{array}{l}\text { Total Sample } \\
\qquad(\mathrm{N}=101)\end{array}$ & $\begin{array}{l}\text { Delirium } \\
(\mathrm{n}=37)\end{array}$ & No Delirium $(n=64)$ & $P$-Value \\
\hline Male, $n(\%)$ & $50(49.5)$ & $15(40.5)$ & $35(54.7)$ & $.22^{*}$ \\
\hline \multicolumn{5}{|l|}{ Education, years, n (\%) } \\
\hline 0 & $20(19.8)$ & $8(21.6)$ & $12(18.7)$ & $.85^{\star}$ \\
\hline $1-4$ & $69(68.3)$ & $24(64.9)$ & $45(70.3)$ & \\
\hline No smoking & $82(81.2)$ & $30(81.1)$ & $52(81.3)$ & $>.99^{*}$ \\
\hline Past or active smoking & $19(18.8)$ & $7(18.9)$ & $12(18.7)$ & \\
\hline \multicolumn{5}{|l|}{ Alcohol, n (\%) } \\
\hline No active drinking & $46(45.5)$ & $24(64.9)$ & $22(34.4)$ & $.004^{*}$ \\
\hline Active drinking & $55(54.5)$ & $13(35.1)$ & $42(65.6)$ & \\
\hline $\begin{array}{l}\text { Charlson Comorbidity Index, mean } \pm \text { SD } \\
\text { (range) }\end{array}$ & $0.5 \pm 0.7(0-4)$ & $0.7 \pm 0.9(0-4)$ & $0.5 \pm 0.6(0-3)$ & $.36^{*}$ \\
\hline Geriatric Depression Scale, mean \pm SD (range) & $4.5 \pm 3.2(0-12)$ & $4.6 \pm 3(0-12)$ & $4.4 \pm 3.2(0-12)$ & $.55^{\ddagger}$ \\
\hline $\begin{array}{l}\text { Number of different medications taken } \\
\text { by the participant outside the hospital during } \\
\text { the week before the surgery and administered } \\
\text { in the hospital until the day before } \\
\text { surgery, mean } \pm \text { SD (range) }\end{array}$ & $3.4 \pm 2.4(0-9)$ & $4.0 \pm 2.8(0-9)$ & $3.0 \pm 2.1(0-9)$ & $.11^{*}$ \\
\hline $\begin{array}{l}\text { Anticholinergic activity of administrated } \\
\text { medications in the week before surgery as } \\
\text { determined by the Anti-cholinergic Drug } \\
\text { Scale }{ }^{20} \text { mean } \pm \text { SD (range) }\end{array}$ & $0.7 \pm 1.1(0-5)$ & $0.8 \pm 1.3(0-5)$ & $0.6 \pm 0.7(0-3)$ & $.7^{\star}$ \\
\hline General anesthesia, $\mathrm{n}(\%)$ & $68(67.3)$ & $29(78.4)$ & $39(60.9)$ & $.08^{*}$ \\
\hline Perioperative transfusion, $\mathrm{n}(\%)$ & $31(30.7)$ & $15(40.5)$ & $16(25)$ & $.08^{*}$ \\
\hline
\end{tabular}

standard curve. Because IL-10 could be measured in all samples, plasma levels of each cytokine were used to calculate the proinflammatory to anti-inflammatory ratio (P/ A) according to the formula (IL-1 $\beta+$ TNF- $\alpha+$ IL- $6+$ IL8)/ IL-10.

\section{Statistical Analysis}

Data were analyzed using the SPSS, version 17.0.2 (PASW Statistics 17, SPSS, Inc., Chicago, IL). The significance of the difference between the pre- and postoperative levels of each biomarker was determined using the Student t-test for paired samples or the Wilcoxon test as a nonparametric alternative. Comparison between participants with and without delirium for pre and postoperative values was performed using the Student t-test for independent samples (normal distributions) or the Mann Whitney U-test (non-normal distributions). The Pearson chi-square test was used to analyze the relationship between categorical variables. The association between preoperative cholinesterase activity and the difference between the two determinations within participants (endpoint - baseline levels, $\Delta$ ) for each inflammatory marker was assessed using the Spearman correlation coefficient. A type I $(\alpha)$ error probability of 0.05 was considered for all inferential analysis.

\section{RESULTS}

\section{Plasma Cholinesterase Activity}

Although surgery induced an overall reduction in AChE and BuChE plasma activity in the control and delirium groups, participants who developed delirium had substantially lower levels of plasma esterase activity before surgery than controls (Table 2).

Table 2. Pre- and Postoperative Activity Levels of Plasma Cholinesterases

\begin{tabular}{|c|c|c|c|c|c|}
\hline \multirow[b]{2}{*}{$\begin{array}{c}\text { Plasma } \\
\text { Cholinesterase }\end{array}$} & \multicolumn{2}{|c|}{ Preoperative } & \multicolumn{2}{|c|}{ Postoperative } & \multirow[b]{2}{*}{$\begin{array}{c}P- \\
\text { Value }\end{array}$} \\
\hline & $\begin{array}{l}\text { Mean } \pm \\
\text { SD }\end{array}$ & $\begin{array}{c}P- \\
\text { Value* }^{*}\end{array}$ & $\begin{array}{c}\text { Mean } \\
\text { SD }\end{array}$ & $\begin{array}{c}P- \\
\text { Value* }^{*}\end{array}$ & \\
\hline \multicolumn{6}{|c|}{ Acetylcholinesterase } \\
\hline Delirium & $2.42 \pm 0.57$ & .01 & $1.88 \pm 0.46$ & .09 & $<.001$ \\
\hline No delirium & $2.71 \pm 0.51$ & & $2.04 \pm 0.45$ & & $<.001$ \\
\hline \multicolumn{6}{|c|}{ Butyrylcholinesterase } \\
\hline Delirium & $4.79 \pm 1.28$ & .04 & $3.24 \pm 0.74$ & .03 & $<.001$ \\
\hline No delirium & $5.33 \pm 1.21$ & & $3.61 \pm 0.84$ & & $<.001$ \\
\hline
\end{tabular}

* Student $t$-test for independent samples.

${ }^{\dagger}$ Student $t$-test for paired samples.

$\mathrm{SD}=$ standard deviation. 


\section{Plasma Levels of Inflammatory Mediators}

Preoperative levels of CRP, IL- $1 \beta$, and TNF- $\alpha$ were below the detection level in $8 \%, 21 \%$, and $27 \%$ of participants, respectively. All participants who developed delirium presented preoperatively with detectable levels of CRP $(0.002 \mathrm{ng} / \mathrm{mL})$, whereas this marker was measurable in $87.5 \%$ of the remaining participants $(P=.02)$. In the postoperative sample, CRP, IL- $1 \beta$, TNF- $\alpha$, and IL- 6 were not measurable in $4 \%, 28 \%, 44 \%$, and $3 \%$ of participants, respectively. These percentages were similar in participants with and without delirium. The proportion of participants with CRP of $5 \mathrm{ng} / \mathrm{mL}$ or greater (a cutoff value used in clinical practice) was similar between the groups with and without delirium before surgery $(10.8 \%$ vs $7.8 \%, P=.72)$ and on the first postoperative day $(78.4 \%$ vs $81.2 \%$, $P=.80)$.

No differences were observed between the two groups regarding plasma levels of each mediator pre- or postoperatively (Table 3). Participants with delirium had a significantly higher P/A ratio on the first postoperative day (Table 3).

\section{Correlation Between Baseline Cholinesterase Activity and Magnitude of Inflammatory Reaction (A)}

Plasma activity of AChE and BuChE before surgery correlated with $\triangle \mathrm{CRP}$ in participants who developed delirium $(\rho=0.428, P=.008$, and $\rho=0.423, P=.009$; Figure $1 \mathrm{~A}$ and $\mathrm{B}$, respectively) but not in controls $(\rho=0.057, P=.65$ and $\rho=0.085, P=.50)$. Similarly, AChE activity correlated with $\Delta \mathrm{IL}-6(\rho=0.339, P=.04$; Figure $1 \mathrm{C})$ and with $\Delta \mathrm{P} / \mathrm{A}$ ratio $(\rho=0.346, P=.04$, Figure $1 \mathrm{D})$ in participants with delirium but not in those who did not developed delirium $(\rho=0.028, P=.82$ and $\rho=0.207, P=.10$, respectively). No correlations were found between baseline plasma cholinesterase activity and within-individual difference in each inflammatory mediator (data not shown).

\section{DISCUSSION}

It has been proposed that central cholinergic dysfunction and a deregulated neuroinflammatory reaction in response to systemic inflammation may be involved in delirium pathophysiology. ${ }^{2,21}$ In addition, the CNS modulates the innate immune response through the cholinergic output of the vagus nerve. ${ }^{3}$ It has recently been reported that individuals with delirium had lower plasma levels of cholinesterase activity than controls even before surgery, suggesting that lower plasma levels of cholinesterase activity may precede and even contribute to the development of an acute confusional state. ${ }^{19}$

The current study demonstrated that, when people were challenged with surgical trauma, production of CRP was significantly higher and that a shift toward a proinflammatory response (as shown by a greater P/A ratio) was observed in patients with delirium. This suggests that an imbalance between proinflammatory and antiinflammatory factors, in favor of a proinflammatory response, might be involved in delirium. Conversely, in agreement with several studies ${ }^{7-14}$ none of the plasma levels of each inflammatory marker measured in this study were related to delirium.

Although these findings are discordant with previous reports of a possible association between inflammatory

Table 3. Plasma Levels of Inflammatory Markers Before and After Surgery and within (Pre- and Postoperative) and Between (Delirium vs No Delirium) Groups

\begin{tabular}{|c|c|c|c|c|c|}
\hline \multirow[b]{2}{*}{ Inflammatory Marker } & \multicolumn{2}{|c|}{ Preoperative } & \multicolumn{2}{|c|}{ Postoperative } & \multirow[b]{2}{*}{$P$-Value } \\
\hline & Median (IQR) & $P$-Value* & Median (IQR) & $P$-Value* & \\
\hline \multicolumn{6}{|l|}{ C-reactive protein } \\
\hline Delirium & $0.38(1.17)$ & .86 & $18.31(11.82)$ & .67 & $<.001$ \\
\hline No delirium & $0.51(0.91)$ & & 15.89 (15.31) & & $<.001$ \\
\hline \multicolumn{6}{|l|}{ IL-1 $\beta$} \\
\hline Delirium & $0.40(0.55)$ & .57 & $0.4(0.39)$ & .86 & .90 \\
\hline No delirium & $0.46(0.61)$ & & $0.4(0.38)$ & & .05 \\
\hline \multicolumn{6}{|l|}{ Tumor necrosis factor alpha } \\
\hline Delirium & $6.12(9.28)$ & .54 & $1.5(5.01)$ & .98 & .50 \\
\hline No delirium & $6.2(9.96)$ & & $1.5(8.3)$ & & .22 \\
\hline \multicolumn{6}{|l|}{ IL-6 } \\
\hline Delirium & $7.95(8.59)$ & .65 & $117.39(148.07)$ & .14 & $<.001$ \\
\hline No delirium & $8.27(8.49)$ & & $93.18(99.25)$ & & $<.001$ \\
\hline \multicolumn{6}{|l|}{ |L-8 } \\
\hline Delirium & $9.16(7.02)$ & .56 & $18.83(17.3)$ & .14 & $<.001$ \\
\hline No delirium & $8.6(6.51)$ & & $16.59(11.25)$ & & $<.001$ \\
\hline \multicolumn{6}{|l|}{ IL-10 } \\
\hline Delirium & $1.81(1.64)$ & .10 & $3.17(3.23)$ & .97 & $<.001$ \\
\hline No delirium & $2(1.37)$ & & $3.18(3.85)$ & & $<.001$ \\
\hline \multicolumn{6}{|l|}{ Pro-/anti-inflammatory ratio } \\
\hline Delirium & $12.44(9.61)$ & .18 & $38.06(27.13)$ & .049 & $<.001$ \\
\hline No delirium & $11.14(5.71)$ & & $28.31(26)$ & & $<.001$ \\
\hline
\end{tabular}



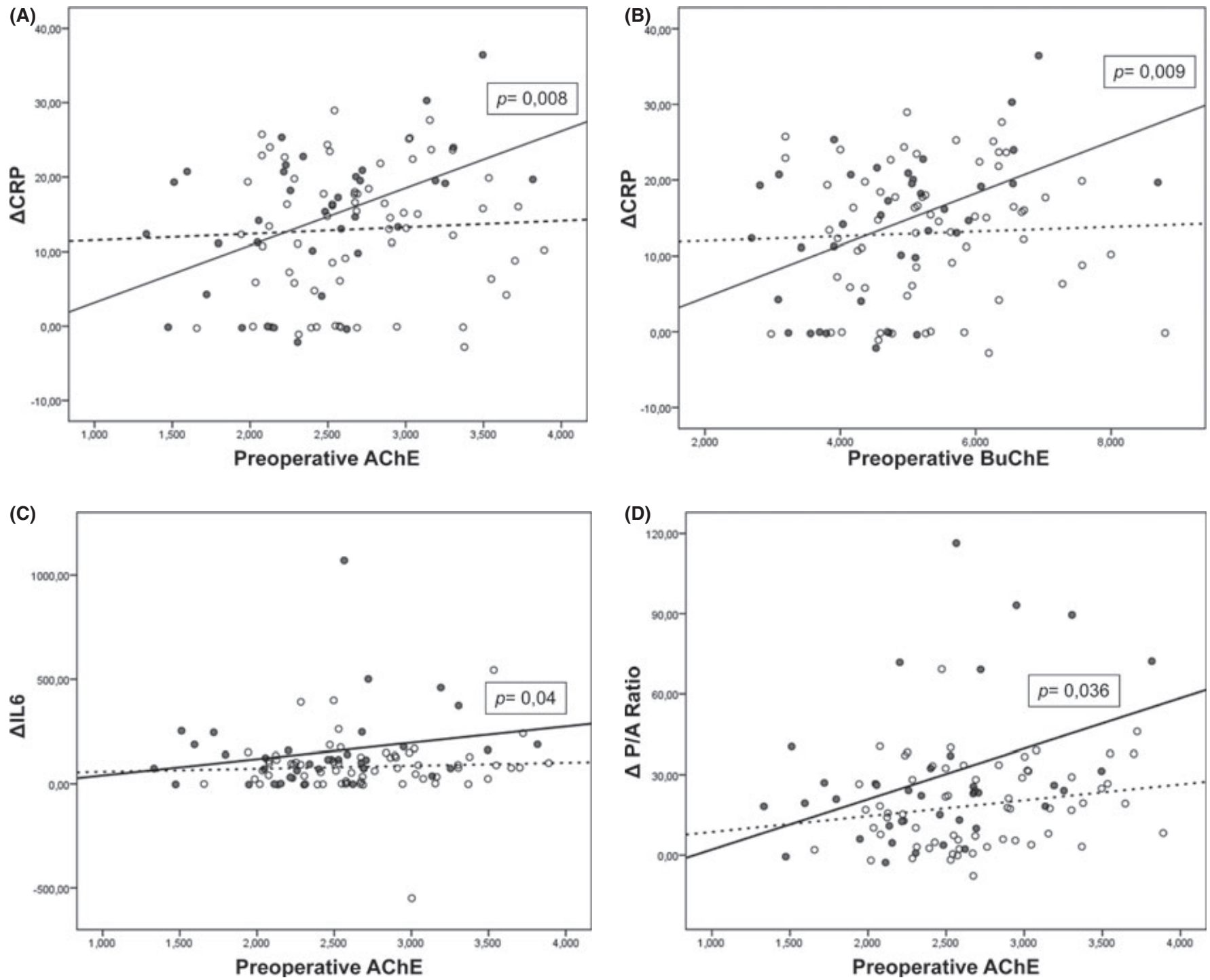

Figure 1. Scatter plots and Spearman correlation coefficients of significant association between preoperative plasma cholinesterase activity and inflammatory markers in participants who developed delirium (०-) and controls $\left(0_{-}--_{-}\right)$. $\Delta=$ end point minus baseline levels; $\mathrm{P} / \mathrm{A}=$ proinflammatory/anti-inflammatory ratio.

markers and delirium ${ }^{4-7,12,15}$ it is likely that isolated levels of cytokines in the bloodstream do not accurately capture the complex homeostatic dysfunction occurring during a delirium episode. This is not surprising, considering that cytokines have pleiotropic and redundant functions within a complex network of various interactions between them. ${ }^{22}$ Consequently, the organism response to acute release of inflammatory mediators into the bloodstream depends more on the integrated actions of overlapping intracellular signaling pathways shared by different pathophysiological routes than on the level of a particular cytokine. P/A ratios might be a more valuable index of the association between systemic cytokine response and relevant clinical outcomes not only in coronary syndromes ${ }^{23,24}$ and sepsis, ${ }^{25,26}$ but also in delirium, as the current results suggest.

A dynamic and intricate cross-talk with the central nervous system and neuroendocrine systems tightly modulates immune system actions, including cytokine production and effects. ${ }^{27}$ In particular, the inhibitory control of the cholinergic anti-inflammatory pathway on the innate immune response is a recently described homeostatic mechanism maintaining proinflammatory responses within an adequate range. ${ }^{3}$ In response to systemic inflammation, the CNS stimulates vagus nerve outflow with the release of acetylcholine. Through inhibition of nuclear factor kappa B nuclear translocation and activation of janus kinase signal transducer and activator of transcription pathways, acetylcholine attenuates the production of TNF, IL-1 $\beta$, IL- 6 , and IL-18 immune cells expressing the nicotinic acetylcholine receptor subunit $\alpha 7 .{ }^{18}$ Under resting conditions, the set point of this reflex arch contributes to establishing the magnitude of the innate inflammatory response to surgical trauma. ${ }^{3}$ Plasma cholinesterase activity, resulting from the conjoined action of peripheral AChE and BuChE, is responsible for the hydrolysis of acetylcholine, thus regulating peripheral cholinergic signaling.

A positive correlation was found between baseline plasma cholinesterase activity and high CRP, IL-6, and 
P/A ratio after surgery in participants with delirium but not in controls. Thus, overactivity of the relationship between cholinergic and inflammatory systems emerges as a common feature in the participants with delirium, in whom exposure to surgery induced different degrees of inflammation according to baseline status of the peripheral cholinergic system (Figure 2). In contrast, the inflammatory response after surgery was independent of baseline cholinesterase activity in participants without delirium. It is plausible that a hyperactive cholinergic anti-inflammatory pathway may underlie the greater strength of correlation between plasma cholinesterase activity and the levels of inflammatory markers observed in individuals with delirium. The lack of correlation in the control group indicates a weaker relationship between the cholinergic and immune systems and the contribution of other factors, not addressed in this study, maintaining the inflammatory response tightly regulated. The detailed mechanisms by which the responsiveness of the cholinergic antiinflammatory pathway is regulated remain unclear. Recent evidence suggests that vagal reactivity and cholinesterase activity depend on the constitutive expression of acetylcholine receptors at the peripheral organs. ${ }^{28}$

The results of the current study indicate that plasma cholinesterase activity can be a candidate biomarker of the inhibitory cholinergic tone at the periphery. When considering the group of participants with delirium, higher baseline cholinesterase activity was associated with heightened proinflammatory response, whereas participants with lower cholinesterase activity before surgery had a lower proinflammatory reaction. A definite conclusion cannot be reached about how $\mathrm{AChE}$ and $\mathrm{BuChE}$ activity levels correlate with peripheral levels of acetylcholine or with the responsiveness of vagal nerve to inflammation, but a dosedependent positive correlation between cholinesterase activity and peripheral cytokine response to immune chal- lenge, largely mediated by the vagus nerve, has been demonstrated. ${ }^{29,30}$ Also, the rate of acetylcholine degradation in peripheral organs critically determines the physiological effects of vagus nerve activity. ${ }^{31,32}$ It is therefore conceivable that lower plasma AChE and BuChE levels are associated with a greater supply of acetylcholine for ligation with cholinergic receptors in immune cells downregulating inflammation (Figure 2). This provides insight into the potential reasons for failure in previous attempts to modulate the cholinergic and inflammatory imbalance through administration of AChE inhibitors. Thus, in a recent randomized controlled trial, critically ill patients with delirium receiving a cholinesterase inhibitor (rivastigmine) had greater mortality than participants not treated with this drug. ${ }^{33}$ Alternatively, recent experimental evidence shows that administration of AChE inhibitors suppresses systemic inflammation and enhances survival of animals exposed to lipopolysaccharides ${ }^{29,30}$ or infection. ${ }^{34}$ These conflicting results demonstrate that any modulation of the cholinergic system should take into account the complex regulatory mechanisms determining the participant's baseline immune set point and the magnitude of responsiveness to inflammatory challenge.

An obvious strength of this study is the use of a prospective design in which several markers were simultaneously measured before and after a controlled traumatic event (hip arthroplasty) in a well-characterized sample of participants with no prior cognitive impairment, although this limits the generalization of the results to clinical samples more likely to benefit from interventions to prevent and treat delirium. The technology used in this study (multiplex arrays) has a number of advantages over traditional enzyme-linked immunosorbent assay (ELISA), offering the possibility of analyzing a panel of cytokines in a cost-effective fashion. Nevertheless, although some studies in delirium have used multiplex technology, ${ }^{8,12,14,15}$ experience with this method

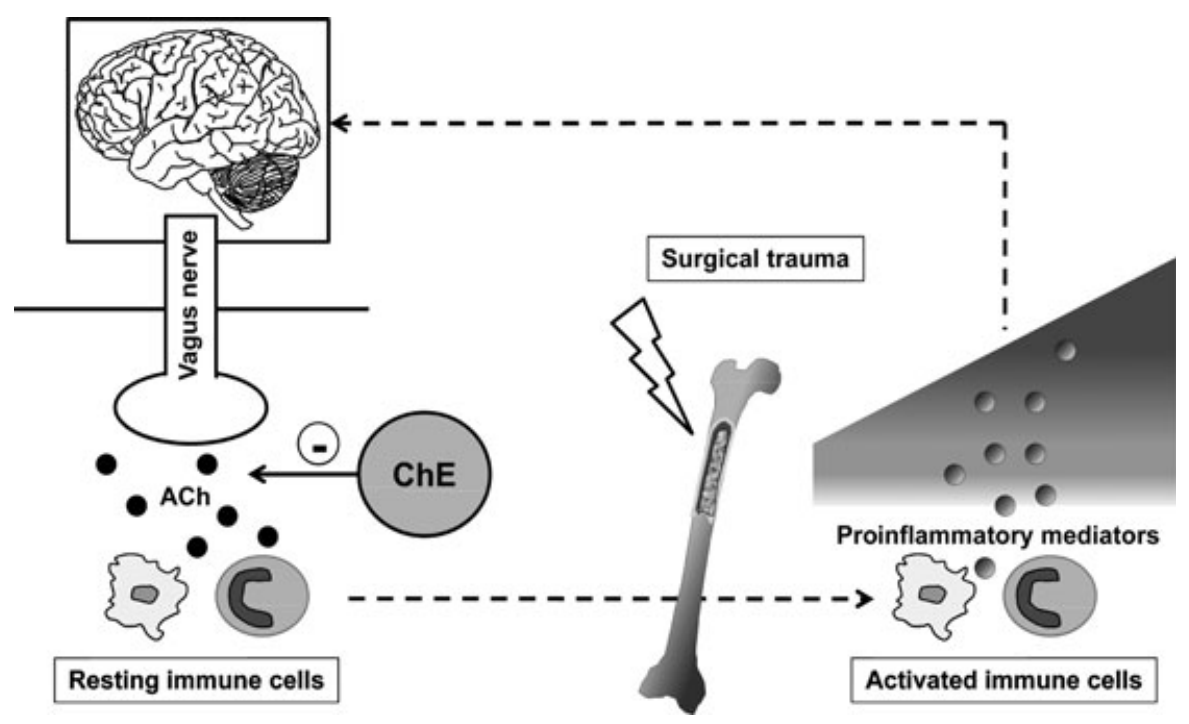

Figure 2. Relationship between the cholinergic and inflammatory systems in delirium. At the periphery, the cholinergic anti-inflammatory pathway modulates the immune system through the interaction of acetylcholine (ACh) with immune cells. In baseline conditions, acetylcholine availability depends on vagus nerve output and its degradation by cholinesterases (ChE). In patients with delirium, exposure to surgical trauma induced different degrees of inflammation according to the baseline status of the peripheral ChE activity, suggesting that delirium is associated with an overactive relation between cholinergic and inflammatory systems. 
remains limited, and comparisons with prior studies using ELISA should be made cautiously because concordance between the two methods has not been definitively established. ${ }^{35}$ Although the assay used in the current study provides a lower limit of detection of IL-1 $\beta$ than previous studies, the levels of this cytokine, as in the case of TNF- $\alpha$, were undetectable in a significant proportion of samples, and no changes were observed after surgery, confirming that these potent proinflammatory cytokines, which have brief half-lives in circulation, are unlikely to be useful markers of acute injury or illness. ${ }^{36}$ Because the postoperative blood draw and the clinical assessment of delirium were not coincident in time, the observed changes in postoperative cytokine levels may not reflect the status of the immune system during the delirium episode.

In conclusion, delirium is associated with an inflammatory imbalance favoring the production of proinflammatory cytokines. Furthermore, changes in the cholinergic system, observed preoperatively, predicted the magnitude of the inflammatory response to surgical trauma. Thus, this study strengthens the view that a comprehensive understanding of the relationship between the cholinergic and immune systems is crucial to yielding new insights into delirium pathophysiology.

\section{ACKNOWLEDGMENTS}

We would like to thank all participants in the study and their families for their support during various stages of this research. We are grateful to the nursing staff and to Miss Maxine Berwick and Mrs. Isabel Amoedo for secretarial support.

Conflict of Interest: The authors declare that they have no competing conflict of interest in relation to the work described.

Author Contributions: Joaquim Cerejeira, Vasco Nogueira, and Pedro Batista: Acquisition of data and preparation of the manuscript. Joaquim Cerejeira, Adriano Vaz-Serra, and Elizabeta B. Mukaetova-Ladinska: Study concept and design and analysis and interpretation of data.

Sponsor's Role: Not applicable.

\section{REFERENCES}

1. Cerejeira J, Mukaetova-Ladinska EB. A clinical update on delirium: From early recognition to effective management. Nurs Res Pract 2011;2011:875196

2. Cerejeira J, Firmino H, Vaz-Serra A et al. The neuroinflammatory hypothesis of delirium. Acta Neuropathol 2010;119:737-754.

3. Tracey KJ. Reflex control of immunity. Nat Rev Immunol 2009;9:418-428

4. Macdonald A, Adamis D, Treloar A et al. C-reactive protein levels predict the incidence of delirium and recovery from it. Age Ageing 2007;36:222-225.

5. White S, Eeles E, O’Mahony S et al. Delirium and C-reactive protein. Age Ageing 2008;37:123-124.

6. Burkhart CS, Dell-Kuster S, Gamberini M et al. Modifiable and nonmodifiable risk factors for postoperative delirium after cardiac surgery with cardiopulmonary bypass. J Cardiothorac Vasc Anesth 2010;24:555-559.

7. Rudolph JL, Ramlawi B, Levkoff SE et al. Inflammation and delirium after cardiac surgery. J Am Geriatr Soc 2006;54(4S):S201-S202.

8. de Rooij SE, van Munster BC, Korevaar JC et al. Cytokines and acute phase response in delirium. J Psychosom Res 2007;62:521-525.

9. Adamis D, Treloar A, Martin FC et al. APOE and cytokines as biological markers for recovery of prevalent delirium in elderly medical inpatients. Int J Geriatr Psychiatry 2007;22:688-694.

10. Adamis D, Lunn M, Martin FC et al. Cytokines and IGF-I in delirious and nondelirious acutely ill older medical inpatients. Age Ageing 2009;38:326-332.
11. Hudetz JA, Patterson KM, Iqbal Z et al. Ketamine attenuates delirium after cardiac surgery with cardiopulmonary bypass. J Cardiothorac Vasc Anesth 2009;23:651-657.

12. van Munster BC, Korevaar JC, Zwinderman AH et al. Time-course of cytokines during delirium in elderly patients with hip fractures. J Am Geriatr Soc 2008;56:1704-1709.

13. Lemstra AW, Kalisvaart KJ, Vreeswijk R et al. Pre-operative inflammatory markers and the risk of postoperative delirium in elderly patients. Int J Geriatr Psychiatry 2008;23:943-948.

14. Rudolph JL, Ramlawi B, Kuchel GA et al. Chemokines are associated with delirium after cardiac surgery. J Gerontol A Biol Sci Med Sci 2008;63A:184-189.

15. van Munster BC, Bisschop PH, Zwinderman $\mathrm{AH}$ et al. Cortisol, interleukins and S100B in delirium in the elderly. Brain Cogn 2010;74:18-23.

16. Holmes C, Cunningham C, Zotova E et al. Proinflammatory cytokines, sickness behavior, and Alzheimer disease. Neurology 2011;77:212-218.

17. Beloosesky Y, Hendel D, Weiss A et al. Cytokines and C-reactive protein production in hip-fracture-operated elderly patients. J Gerontol A Biol Sci Med Sci 2007;62A:420-426.

18. Rosas-Ballina M, Tracey KJ. Cholinergic control of inflammation. J Intern Med 2009;265:663-679

19. Cerejeira J, Batista P, Nogueira V et al. Low preoperative plasma cholinesterase activity as a risk marker of postoperative delirium in elderly patients. Age Ageing 2011;40:621-626.

20. Carnahan RM, Lund BC, Perry PJ et al. The Anticholinergic Drug Scale as a measure of drug-related anticholinergic burden: Associations with serum anticholinergic activity. J Clin Pharmacol 2006;46:1481-1486.

21. Hshieh TT, Fong TG, Marcantonio ER et al. Cholinergic deficiency hypothesis in delirium: A synthesis of current evidence. J Gerontol A Biol Sci Med Sci 2008;63A:764-772.

22. Lin E, Calvano SE, Lowry SF. Inflammatory cytokines and cell response in surgery. Surgery 2000;127:117-126.

23. Kilic T, Ural D, Ural E et al. Relation between proinflammatory to antiinflammatory cytokine ratios and long-term prognosis in patients with nonST elevation acute coronary syndrome. Heart 2006;92:1041-1046.

24. Rajappa M, Sen SK, Sharma A. Role of pro-/anti-inflammatory cytokines and their correlation with established risk factors in South Indians with coronary artery disease. Angiology 2009;60:419-426.

25. Kellum JA, Kong L, Fink MP et al. Understanding the inflammatory cytokine response in pneumonia and sepsis: Results of the Genetic and Inflammatory Markers of Sepsis (GenIMS) Study. Arch Intern Med 2007;167:1655-1663.

26. Gogos CA, Drosou E, Bassaris HP et al. Pro- versus anti-inflammatory cytokine profile in patients with severe sepsis: A marker for prognosis and future therapeutic options. J Infect Dis 2000;181:176-180.

27. O'Connor JC, McCusker RH, Strle K et al. Regulation of IGF-I function by proinflammatory cytokines: At the interface of immunology and endocrinology. Cell Immunol 2008;252:91-110.

28. Livolsi A, Niederhoffer N, Dali-Youcef $\mathrm{N}$ et al. Constitutive overexpression of muscarinic receptors leads to vagal hyperreactivity. PLoS ONE 2010;5:e15618. Erratum in: PLoS One 2011;6. doi: 10.1371/annotation/ 2747fb4d-de47-4e66-81f9-76b5f5ac4b90.

29. Pavlov VA, Parrish WR, Rosas-Ballina $M$ et al. Brain acetylcholinesterase activity controls systemic cytokine levels through the cholinergic antiinflammatory pathway. Brain Behav Immun 2009;23:41-45.

30. Liu ZH, Ma YF, Wu JS et al. Effect of cholinesterase inhibitor galanthamine on circulating tumor necrosis factor alpha in rats with lipopolysaccharide-induced peritonitis. Chin Med J (Engl) 2010;123:1727-1730.

31. Nakahara T, Kawada T, Sugimachi M et al. Cholinesterase affects dynamic transduction properties from vagal stimulation to heart rate. Am J Physiol 1998;2:R541-R547.

32. Paes AM, Carniatto SR, Francisco FA et al. Acetylcholinesterase activity changes on visceral organs of VMH lesion-induced obese rats. Int J Neurosci 2006;116:1295-1302.

33. van Eijk MM, Roes KC, Honing ML et al. Effect of rivastigmine as an adjunct to usual care with haloperidol on duration of delirium and mortality in critically ill patients: A multicentre, double-blind, placebo-controlled randomised trial. Lancet 2010;376:1829-1837.

34. Fernandez-Cabezudo MJ, Lorke DE, Azimullah S et al. Cholinergic stimulation of the immune system protects against lethal infection by Salmonella enterica serovar Typhimurium. Immunology 2010;130:388-398.

35. Leng SX, McElhaney JE, Walston JD et al. ELISA and multiplex technologies for cytokine measurement in inflammation and aging research. J Gerontol A Biol Sci Med Sci 2008;63A:879-884.

36. Giannoudis PV. Current concepts of the inflammatory response after major trauma: An update. Injury 2003;34:397-404. 